\title{
Chromosome Microarray and Undiagnosed Seizures in a Pediatric Patient
}

\author{
Angelika J. Dawson, Aizeddin A. Mhanni, Frances A. Booth, Lorne Seargeant, Daniele Bernier, \\ Michelle Tomiuk, Jessica N. Hartley, Michelle Strecker, Karine Hovanes
}

Can J Neurol Sci. 2014; 41: 281-283

Chromosome microarrays have revolutionized conventional cytogenetics due to their greatly increased resolution, resulting in a greater number of chromosome abnormalities detected. Chromosome microarray analysis is the recommended first tier diagnostic test for children with mental handicap, congenital anomalies/dysmorphisms and autism ${ }^{1}$ and results in a much higher diagnostic yield (15\%-20\%) than a conventional G-band karyotype (approximately 3\%, excluding Down syndrome and other recognizable chromosomal syndromes) ${ }^{1}$.

Numerous conventional chromosome disorders, such as the Wolf-Hirschhorn (4p-), Angelman (15q-), Miller-Dieker (17p-), Klinefelter (XXY), and Down (+21) syndromes, have been reported in association with various neurological disorders, including seizures and epilepsy ${ }^{2}$. However, many children with a neurological disorder, such as severe epilepsies, have a normal G-band karyotype with no diagnosis, although a genetic etiology is often suspected ${ }^{3}$. Chromosome microarray has recently been reported to detect clinically significant chromosome abnormalities in approximately $9 \%$ of patients with a broad range of neurologic phenotypes of unknown etiology, including severe epilepsy ${ }^{3}$. For example, a variety of seizures types were reported in association with the newly identified $15 q 13.3$ microdeletion syndrome, detectable only by microarray ${ }^{2}$.

A conventional G-band karyotype will not reliably detect deletions or duplications smaller than approximately $5 \mathrm{Mb}$ (megabases). Chromosome microarray allows for the detection of unbalanced DNA copy number variations or changes, i.e. deletions and duplications, at resolutions much less than $1 \mathrm{Mb}$, with the lower limits of resolution in the hundreds of $\mathrm{Kb}$ (kilobases) at gene rich regions. DNA segments from across all chromosomes are used as substitutes for metaphase chromosomes, analogous to a molecular karyotype ${ }^{1}$. In addition, approximately $40 \%$ of patients with a clinical phenotype and an apparently balanced translocation by conventional cytogenetics have been shown, by chromosome microarray, to carry a cryptic imbalance that would be consistent with their clinical phenotype $^{4}$. In prenatal diagnosis, therefore, chromosome microarray diagnosis is recommended for fetuses with a de novo chromosome rearrangement ${ }^{5}$.

We report an apparently balanced, de novo translocation detected prenatally by conventional karyotype in a patient with epilepsy and neurodevelopmental dysfunction of unknown etiology. A postnatal chromosome microarray revealed a cryptic microdeletion at one of the translocation breakpoints. The results of a prenatal microarray would have suggested consideration of the diagnosis and altered management. We report this as an example of the usefulness of early microarray analysis in children with epilepsy of unknown etiology.

\section{Clinical History}

An eight month-old boy presented with recurrent seizures with his first event of a tonic-clonic, afebrile seizure occurring at five months-of-age. He was the first born to a nonconsanguineous healthy 35-year-old mother and 41-year-old father. The patient has a brother and paternal half sister, both of whom are clinically normal. Prenatal and perinatal histories were unremarkable. The description of subsequent seizures varied and included episodes of arching, staring, twitching, eye rolling, unresponsiveness, cyanosis and collapse in various combinations. Periods of apparent regression (increased difficulty with gait and speech) were followed by recovery of these skills. Several episodes of sleepiness unassociated with hypoglycemia but seemingly improving with a "sweet drink" were described shortly before the diagnosis was established. Seizures were not controlled despite multiple anti-epileptic medications. Physical examination revealed microcephaly with an OFC (Occipital Frontal Circumference) of less than the 5th centile; and weight and height, both at approximately the 25 th centile. Neuroimaging, including MRS (magnetic resonance spectroscopy), was non-diagnostic.

\section{BiochemiCAL AND CyTOGENETIC INVESTIGATIONS}

An extensive biochemical work-up on plasma, urine, cerebrospinal fluid and whole blood was performed. Prenatal and postnatal cytogenetic analysis was performed according to standard protocols, at a band resolution of 450 to 550 bands per haploid genome, respectively (Figure 1). Chromosome

\footnotetext{
From the Cytogenetic Laboratory (AJD), Biochemistry Laboratory (LS), Diagnostic Services of Manitoba; Departments of Biochemistry \& Medical Genetics and Pediatrics \& Child Health (AJD, AAM, LS, JNH,), Section of Pediatric Neurology, Departments of Pediatrics \& Child Health (FAB), University of Manitoba; Genetics \& Metabolism Program (AJD, AAM, JNH), WRHA; Cytogenetics (DB, MT), Diagnostic Services of Manitoba, Winnipeg, MB, Canada; CombiMatrix Diagnostics (MS, KH), Irvine, California, USA.

Received June 12, 2013. Final Revisions Submitted September 17, 2013 Correspondence to: A.J. Dawson, 820 Sherbrook St. MS543H, Winnipeg, Manitoba, R3A 1R9, Canada. Email: adawson@dsmanitoba.ca.
} 


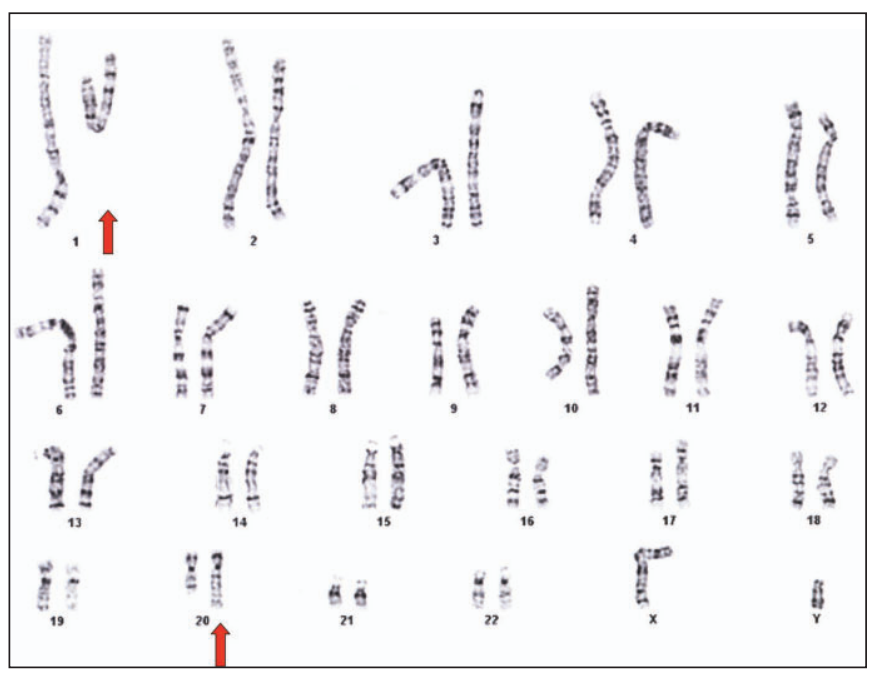

Figure 1: G-banded karyogram of the patient showing the $t(1 ; 20)$ (p34.1;q13.1)dn (red arrows).

microarray analysis was performed at CombiMatrix Diagnostics (CMDx, Irvine, CA) using the oligonucleotide $180 \mathrm{~K}$ microarray (NCBI build 36, March 2006) (Figure 2). All results were confirmed by fluorescent in situ hybridization (FISH) analysis.

\section{RESULTS}

An amniocentesis had been performed because of advanced maternal age (AMA). A de novo, apparently balanced
Table: Oligonucleotide chromosomal microarray analysis

Loss of 1p34.2p34.1 (42,871,391-44,074,069)

[Genes (from centromere to telomere: CCDC30 (partial), PPIH, YBX1, CLDN19, LEPRE1, C1orf50, LOC100129924, CCDC23, ERMAP, ZNF691, SLC2A1, SLC2A1-AS1, FAM183A, EBNA1BP2, WDR65, TMEM125, C1orf210, TIE1, MPL, CDC20, ELOVL1, MED8, SZT2, HYI, PTPRF, KDM4A, KDM4A-AS1, ST3GAL3 (partial)]

The translocation breakpoint on the $\operatorname{der}(20) \mathrm{t}(1 ; 20)$ could not be determined by this assay as it did not involve a copy number change.

translocation was found: $46, \mathrm{XY}, \mathrm{t}(1 ; 20)(\mathrm{p} 34.1 ; \mathrm{q} 13.1) \mathrm{dn}$. The karyotype was repeated at the child's first clinic presentation, with the same conclusion of a balanced, reciprocal translocation (Figure 1). Metabolic work-up revealed a cerebrospinal fluid (CSF) to blood glucose ratio of 0.378 (normal ratio: $0.65+/$ 0.01 ), suggesting a biochemical diagnosis of glucose transporter type 1 (GLUT1) deficiency syndrome (De Vivo disease). The results of the chromosome microarray indicated a $1.2 \mathrm{Mb}$ microdeletion of the $1 \mathrm{p} 34.2$-p34.1 region of the $\operatorname{der}(1) \mathrm{t}(1 ; 20)$ chromosome, including the SLC2A1 gene (Table): arr 1p34.2p34.1(42,871,391-44,074,069)x1. This cryptic microdeletion is below the level of classic cytogenetic resolution (approximately $5 \mathrm{Mb}$ ). The microdeletion was confirmed by

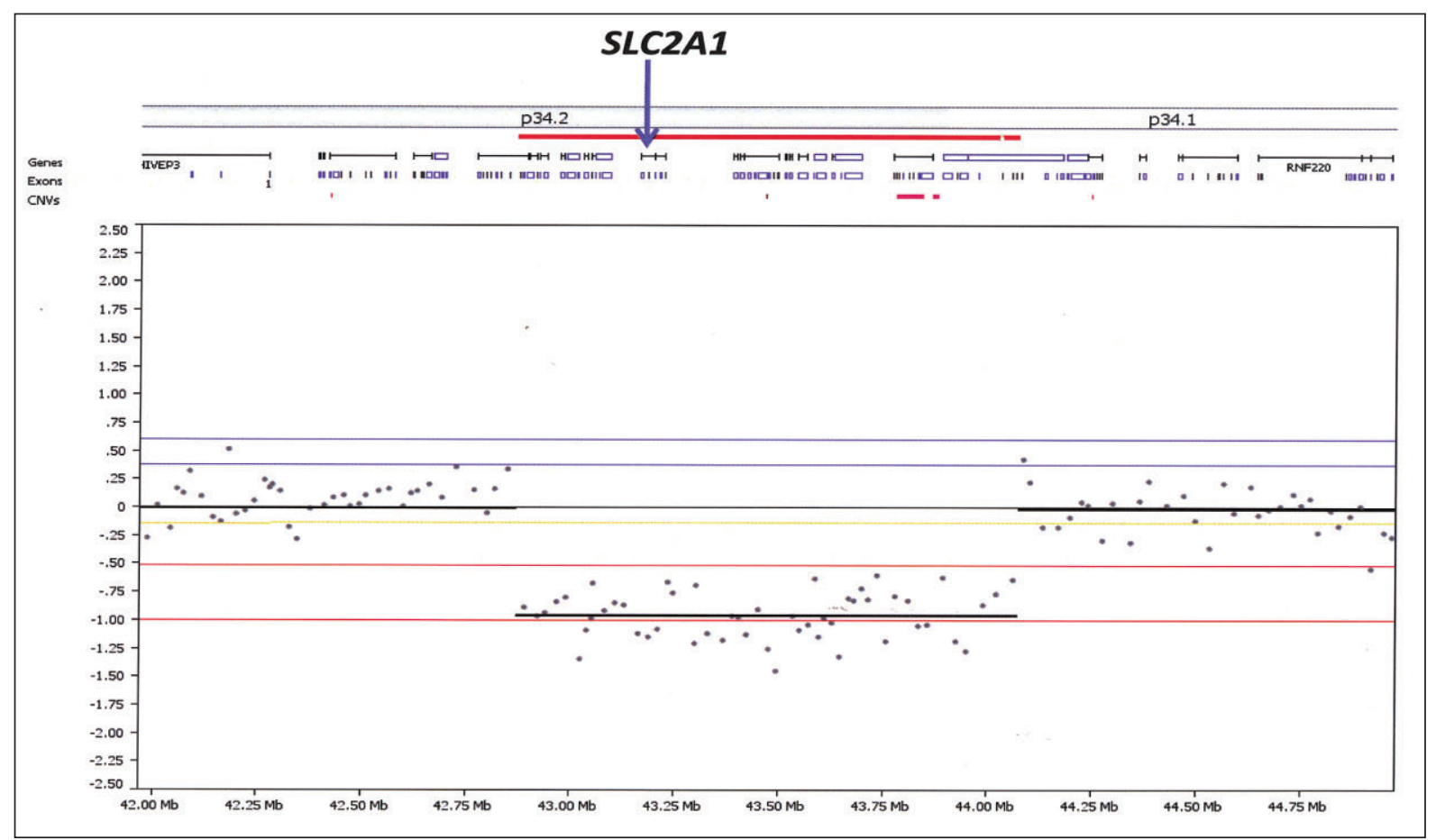

Figure 2: Oligonucleotide 180K microarray analysis showing a 1.2 Mb microdeletion of the 1p34.2-p34.1 region (red line) of the der(1)t(1;20), including the SLC2A1 gene (blue arrow). 
FISH analysis with probes corresponding to loci mapped to 1p34.2 (data not shown). Parental karyotype and microarray analyses revealed normal results (data not shown).

\section{Discussion}

The patient's 550 G-band prenatal and postnatal karyogram appeared to have a balanced, reciprocal translocation: 46,XY,t(1;20)(p34.1;q13.1)dn. Microarray analysis was not performed prenatally at the time as it was not available for prenatal samples at that time. The postnatal chromosome microarray revealed a cryptic, $1.2 \mathrm{Mb}$ microdeletion of 1p34.2->p34.1, which was confirmed by FISH analysis. Parental karyotypes and microarray analysis were normal, confirming that the microdeletion was de novo. The deletion is the result of an unbalanced translocation between $1 \mathrm{p}$ and 20q; and includes OMIM genes SLC2A1, CLDN19, LELPRE1, and MPL (Table). Heterozygous mutations and deletions of the $S L C 2 A 1$ gene are responsible for GLUT1 deficiency, an autosomal dominant disorder characterized by infantile seizures refractory to anticonvulsants, decelerated head growth, mental and motor developmental delay, with variable degrees of cognitive impairment. The three other OMIM genes (CLDN19, LEPRE1, $M P L)$ are associated with autosomal recessive disorders. The translocation breakpoint on $20 \mathrm{q}$ could not be determined by this microarray since it did not involve any copy number changes.

The patient has been on a ketogenic diet for the past two years. He has generally been well and has been making considerable developmental progress. His seizures remain under good control and his head growth is well maintained. This case clearly demonstrates the value of performing chromosome microarray analysis in the investigation of children with developmental delay and epilepsy by providing increased resolution to detect cytogenetic rearrangements, which lead to correlation of clinical manifestations with specific changes in gene copy number ${ }^{1}$. Specifically, in our patient, chromosome microarray analysis detected a condition that was treatable. Furthermore, a prenatal microarray to confirm the apparently balanced, de novo $\mathrm{t}(1 ; 20)$ detected by conventional karyotype would have led to a specific diagnosis of GLUT1 deficiency and altered management from birth.

\section{Conclusions}

Approximately $40 \%$ of reciprocal, cytogenetically balanced de novo translocations associated with abnormal phenotypes have a microdeletion at either one of the breakpoints or elsewhere and should be followed up by chromosome microarray ${ }^{4}$. The prenatal detection of apparently balanced, de novo chromosome rearrangements by conventional cytogenetic techniques should necessarily be followed up with microarray analysis to evaluate the possibility of an imbalance ${ }^{5}$. Chromosomal microarray is now a recommended fist tier clinical test for the evaluation of individuals with developmental delay or congenital anomalies ${ }^{1}$. Notably, in our patient, chromosomal microarray analysis identified not only the etiology of the disease, but also a specific treatment, which resulted in an improved clinical outcome for our patient. We therefore support the recommendation that chromosome microarray should also be a first tier diagnostic test in patients with severe epilepsy, neurologically abnormal neonates and patients with neurological disorders of unknown etiology ${ }^{3}$.

\section{REFERENCES}

1. Miller DT, Adam MP, Aradhya S, et al. Consensus statement: chromosomal microarray is a first-tier clinical diagnostic test for individuals with developmental disabilities or congenital anomalies. Am J Hum Genet. 2010;86:749-64.

2. Sorge G, Sorge A. Epilepsy and chromosomal abnormalities. Italian J Ped. 2010;36:36-43.

3. Howell KB, Kornberg AJ, Harvey AS, Ryan MM, Mackay MT, Freeman JL. High resolution chromosomal microarray in undiagnosed neurological disorders. J Paediatr Child Health. 2013;49:716-24.

4. Schluth-Bolard C, Delobel B, Sanlaville D, et al. Cryptic imbalances in de novo and inherited apparently balanced chromosomal rearrangements: Array CGH study of 47 unrelated cases. Eur J Med Genet. 2009;52:291-6.

5. Chen $\mathrm{CP}, \mathrm{Wu} \mathrm{PC}, \mathrm{Lin} \mathrm{CJ}$, et al. Balanced reciprocal translocations detected at amniocentesis. Taiwan J Obstet Gynecol. 2010;49: 455-67. 